\title{
Propuesta de validación parcial de modelos de simulación de crecimiento urbano basados en autómatas celulares mediante análisis de sensibilidad
}

\author{
Pablo Barreira González, \\ Francisco Aguilera Benavente y \\ Montserrat Gómez Delgado
}

\section{Resumen}

La geosimulación de los procesos urbanos ha cobrado especial importancia en los últimos años, como herramienta para desarrollar metodologías y procedimientos que nos permitan estimar el impacto que el crecimiento urbano provocará en el futuro. Concretamente, los modelos basados en autómatas celulares (AC) son los que más difusión han tenido, dada su capacidad para reproducir la tendencia y las características intrínsecas de estas dinámicas. En el presente trabajo se presenta una alternativa a esta forma tendencial de entender la simulación, utilizando estos modelos para desarrollar alternativas de futuro (escenarios). Los resultados obtenidos, sin embargo, no pueden validarse de forma total, dado que no contamos con datos reales de futuro con los que contrastarlos. En este contexto, se presenta un modelo de simulación de crecimiento urbano basado en AC para la Comunidad de Madrid, que pretende simular un escenario futuro de sostenibilidad. La aportación de este trabajo consistirá en realizar propuestas alternativas de validación parcial del modelo para dar mayor robustez a unos resultados que no pueden ser contrastados con datos reales y así reducir el riesgo asociado a la toma de decisiones sobre la urbanización del territorio.

Palabras clave: Simulación crecimiento urbano, autómatas celulares, validación parcial, análisis de sensibilidad, Comunidad Autónoma de Madrid.

\section{Abstract}

In recent years, geosimulation of urban processes has become important as a tool to develop methods and procedures that allow us to estimate the future impact of urban growth. In particular, cellular automata (CA) based models have been aired 
more than others due to their ability to reproduce tendencies and the intrinsic characteristics of that dynamism. In this work, we present an alternative to this way of simulation, using these models to develop future alternatives (scenarios). Nevertheless, the obtained results cannot be totally validated because there are no real future data to contrast with. In this context, we present an urban growth CA based model for the Madrid Community where a sustainable future scenario is expected to be simulated. The contribution of this work consists of making alternative proposals for the model's partial validation to give more robustness to results that cannot be contrasted with real data and then, reduce the associated risk with the landscape urbanization decision making.

Keywords: Urban growth simulation, cellular automata, partial validation, sensibility analysis, Madrid Region.

Pablo Barreira González (pablobarreiragonzalez@hotmail.com); Francisco Aguilera Benavente (f.aguilera@uah.es); Montserrat Gómez Delgado (montserrat.gomez@uah. es). Departamento de Geografía y Geología, Universidad de Alcalá, Madrid, España. 


\section{INTRODUCCIÓN}

En la actualidad, los cambios de usos del suelo en general, y el crecimiento de los usos urbanos en particular, son dos de los fenómenos más estudiados debido a sus consecuencias sobre el cambio global del planeta. La estimación del impacto de dicho crecimiento y la aplicación de instrumentos de ordenación urbana son de relevante interés para minimizar los daños ocasionados por el desarrollo urbano sobre el entorno, tanto en ámbitos urbanos como rurales (Barredo et al., 2004), procurando así un crecimiento más sostenible.

Es en este contexto donde la combinación de diferentes técnicas, ciencias y metodologías son necesarias para armonizar el desarrollo del territorio. Y es aquí donde la Geosimulación ha mostrado un amplio potencial en este aspecto, ya que permite diseñar y construir modelos espaciales basados en objetos, con el fin de explorar ideas e hipótesis sobre cómo operan los sistemas espaciales (Benenson y Torrens, 2004), combinando elementos de la Matemática Fundamental con la computación, la teoría general de sistemas y evidentemente la Geografía, materializada en su vertiente más actual: las Tecnologías de la Información Geográfica (TIG).

En este sentido, ha surgido una corriente (muy extendida en las últimas décadas) dedicada a la simulación de dinámicas de los usos del suelo. Este tipo de estudios tienen como eje principal los instrumentos de simulación de procesos urbanos, los cuales aportan información de carácter relevante sobre lo que puede ocurrir en un territorio y su posible evolución (Santé et al., 2010), sobre los cambios en los patrones de crecimiento de las ciudades y áreas metropolitanas (Berling-Wolf y Wu, 2004), y cómo estos afectan al paisaje y el territorio donde tienen lugar (Aguilera y Talavera, 2009).

Dentro de la variedad de modelos de simulación de crecimiento urbano disponibles, los modelos basados en autómatas celulares (AC) han sido los más utilizados en las dos últimas décadas para la simulación prospectiva (Batty et al., 1997; White et al., 1997; Barredo et al. 2003; Aguilera et al., 2010; Santé et al., 2010), dada su capacidad para reproducir los comportamientos complejos propios del crecimiento urbano (Torrens, 2000).

Tradicionalmente estos modelos han tenido un enfoque meramente tendencial, siguiendo una metodología similar a la que se muestra en la figura 1. Ésta asume que, tras la calibración del modelo en un espacio de tiempo pasado, será 
capaz de reproducir dinámicas pasadas, como demuestra una validación total. Una vez validado el modelo, se proyecta la tendencia observada hacia el futuro.

Figura 1. Proceso de simulación con modelos basados en AC.

La validación se realiza antes de las simulaciones futuras.

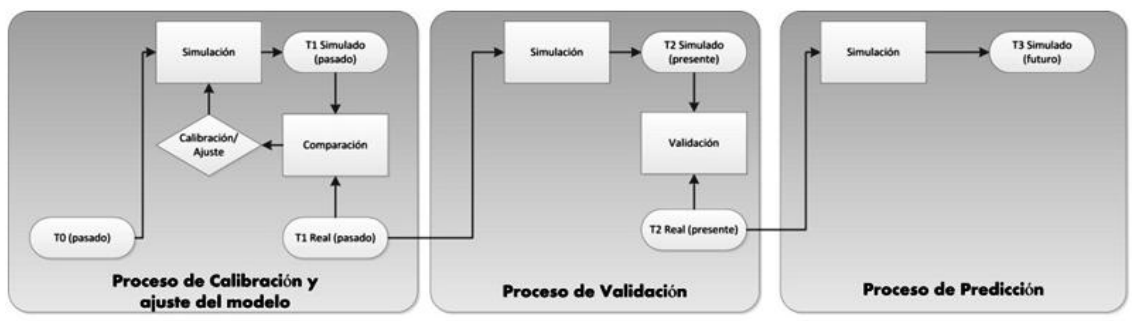

En el caso de la simulación del crecimiento urbano, este enfoque predictivo puede resultar algo problemático (Berdoulay, 2009), dado el grado de incertidumbre que lleva asociado este proceso, dependiendo muchas veces de decisiones político-administrativas o situaciones económicas que divergen de las proyectadas.

Por ello, además de poder simular tendencias pasadas y proyectarlas al futuro, es necesario valorar otro tipo de alternativas para mejorar la planificación urbana. Estas alternativas se presentan a través de la generación de escenarios y su integración dentro de los modelos de simulación de crecimiento urbano (Dreborg, 2004). De esta forma se consigue que la planificación pueda ser más efectiva, reduciendo la incertidumbre asociada al fenómeno de crecimiento urbano. El empleo de esta nueva visión debe desembocar en una modificación del proceso de creación de los modelos de simulación de crecimiento urbano, tal y como recoge la figura 2.

Figura 2. Propuesta de simulación-validación para la exploración de escenarios futuros.

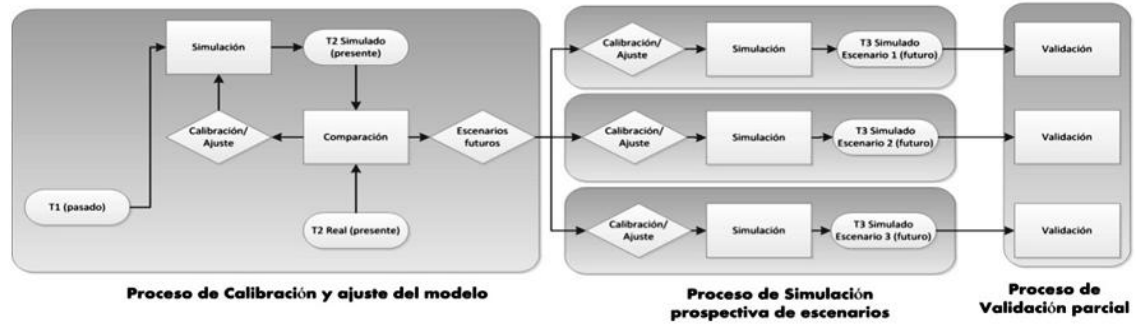


En este caso, tras la calibración del modelo, éste se podría ajustar tantas veces como escenarios futuros sean planteados y es aquí donde surge la necesidad de realizar una propuesta diferente para llevar a cabo su validación. Hemos de tener en cuenta que no se dispondrá nunca de una verdad terreno con la que validar nuestros resultados, por lo que resulta imprescindible desarrollar procedimientos que nos permitan testar de algún modo la fiabilidad y robustez de los resultados de cada uno de los escenarios que se planteen.

Uno de los procedimientos considerados más importantes en cualquier proceso de validación es la evaluación de la estabilidad y robustez de los resultados a través de un análisis de sensibilidad (Qureshi, 1999, Paegelow y Camacho, 2008, Gómez Delgado y Bosque Sendra, 2009). El análisis de sensibilidad (AS) nos permite estudiar cómo la variación de los resultados puede ser atribuida (cualitativa o cuantitativamente) a la variación de las variables y parámetros de partida del modelo (Saltelli et al, 2000). Los resultados de este análisis pueden indicarnos, entre otros aspectos, qué partes del modelo deberían ser mejoradas o a qué datos de partida deberíamos prestar especial atención por su influencia en los resultados (Saltelli, et al., 2008).

Por otro lado, pese al enfoque que tradicionalmente se ha llevado a cabo estudiando la influencia de parámetros implicados en el modelo de forma específica (Ménard y Marceau, 2005; Kocabas y Dragicevic, 2006; García et al., 2011; Pan et al., 2011), se entiende que se debe valorar el conjunto de parámetros y además estudiar qué influencia tiene cada uno en los resultados obtenidos. Para poder estudiar esta influencia, y al tratarse de modelos explícitamente espaciales, es necesario emplear métodos de comparación de mapas. Existen métodos diversos, desde los más tradicionales como la comparación visual y uso de índices estadísticos (Kappa, por ejemplo), hasta otros métodos más novedosos que buscan eliminar la rigidez de la comparación píxel a píxel.

En el presente trabajo se utiliza un AS como procedimiento de validación parcial de simulaciones futuras. Concretamente se aplicará sobre un modelo de simulación de crecimiento urbano basado en AC, desarrollado en la Comunidad Autónoma de Madrid, dentro del proyecto de investigación SIMURBAN ${ }^{1}$. En se-

\footnotetext{
${ }^{1}$ SIMURBAN: análisis y simulación prospectiva mediante Tecnologías de la Información Geográfica (TIG) del crecimiento urbano actual. Evaluación de sus sostenibilidad. (SEJ2007-66608-C04-00/GEOG). Es posible encontrar más información en www.geogra.uah.es/simurban/.
} 
gundo lugar, se pretenden explorar varios métodos de comparación de mapas, herramienta imprescindible para evaluar de manera explícitamente espacial los resultados del AS. Así, una vez planteados diversos modelos derivados del inicial, los resultados serán evaluados a partir de métodos tanto tradicionales, como otras propuestas más novedosas. La última aportación consistirá en la elaboración de un mapa que registre las zonas más veces seleccionadas por los modelos para el desarrollo urbano, pues estas zonas serán las más robustas y sobre las que el proceso de planificación debería prestar especial atención.

\section{2. ÁREA DE ESTUDIO Y MATERIALES}

\subsection{La Comunidad de Madrid.}

La Comunidad de Madrid (figura 3) se ha convertido en uno de los lugares más dinámicos de Europa en las últimas décadas, en lo que a crecimiento urbanos se refiere (Plata Rocha et al., 2010). El aumento de la densidad de población, y en definitiva el crecimiento en número de habitantes per se, constituye uno de los motores activos de la Comunidad de Madrid, alcanzando en 2011 los 6,3 millones de habitantes. Sin embargo, ha sido el contexto económico positivo que experimentó España a finales de los 90 y principios de este milenio (que supuso un aumento de la construcción residencial, la actividad comercial e industrial y que derivó en burbuja inmobiliaria (Burriel, 2011), lo que ha provocado un aumento notable e innecesario del suelo urbano. Este dinamismo se ha traducido, además, en cambios en los patrones de crecimiento urbano con importantes consecuencias sobre la estructura urbana (OSE, 2006).

Aunque actualmente el crecimiento urbano se ha frenado ante el impacto de la crisis económica (Burriel, 2011), la Comunidad de Madrid sigue siendo un territorio interesante para el estudio de las dinámicas urbanas, y especialmente para su simulación contemplando diferentes escenarios a futuro, pues es necesaria una planificación sostenible del territorio que todavía permanece sin urbanizar. 
Figura 3. Situación de los usos urbanos en la Comunidad de Madrid, año base del modelo basado en AC: 2000.

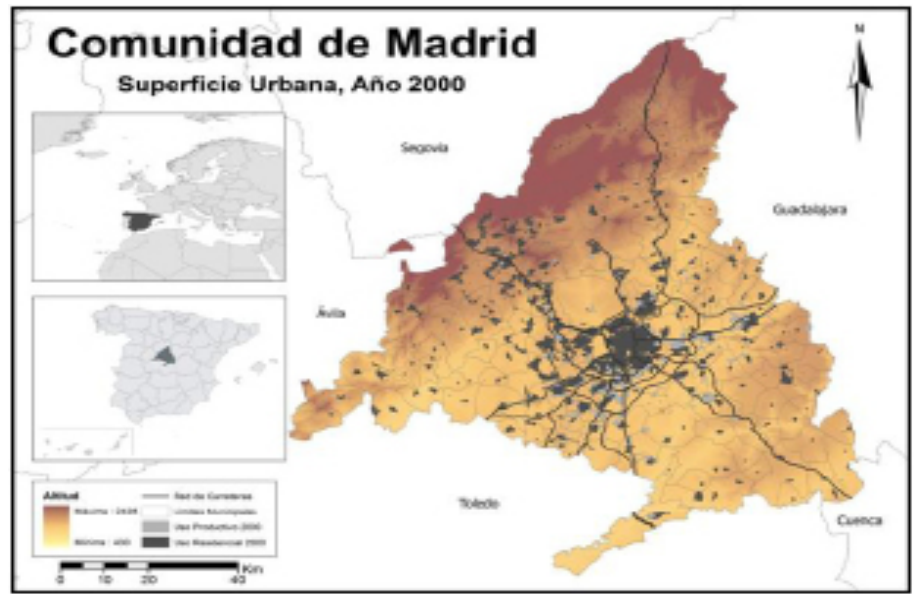

\subsection{El modelo basado en AC.}

En la última década, los modelos basados en $\mathrm{AC}$ han seguido las premisas expuestas por White et al. (1997). Estos modelos representan de forma discreta tanto el espacio (representación ráster), como el tiempo (Barredo et al.,2003, Aguilera,2006; Petrov et al., 2009).

Para el presente trabajo se ha empleado como modelo una modificación del diseñado para simular diferentes escenarios de crecimiento urbano en la Aglomeración Urbana de Granada (Aguilera et al., 2010), adaptado a las características específicas de la Comunidad de Madrid. Este modelo, calibrado con los datos del periodo 1990-2000 para el área de estudio, simula el crecimiento de los usos residencial y productivo (superficies comerciales e industriales) entre los años 2000 y 2020, con un tamaño de píxel de $200 \mathrm{~m}$ de resolución.

El funcionamiento del modelo se rige por las siguientes pautas: para cada lugar del ámbito de estudio (píxel) se estima un potencial de transición (Pj) para cada uno de los usos simulados, de forma que en cada iteración del modelo se tienden a trasformar en zonas urbanas los píxeles con mayor potencial de transición. Este potencial se calcula a través de la combinación de los cuatro factores habitualmente presentes en este tipo de modelos de simulación: vecindad, accesibilidad, aptitud y un parámetro aleatorio. 
El factor vecindad estima la influencia (atracción o repulsión) que tiene en cada píxel los usos que tengan los píxeles vecinos, de forma que, por ejemplo, evite la localización de nuevas zonas industriales muy próximas a zonas residenciales existentes. Por otro lado la aptitud pretende determinar la capacidad que tiene cada píxel para albergar un uso urbano, dadas sus características geográficas: pendiente, altitud, distancia a hidrografía, etc. La accesibilidad calcula para cada píxel la capacidad de acceso a la red viaria y a otros equipamientos y servicios básicos. Finalmente el modelo cuenta con un parámetro de aleatoriedad o estocástico que pretende reproducir la incertidumbre con la que se producen los crecimientos urbanos.

Este modelo posee la flexibilidad necesaria para simular diversos escenarios, siendo además adaptable a la representación de diferentes patrones o formas de crecimiento (Aguilera et al, 2011). En el caso de la propuesta de AS planteada, se empleará uno de los escenarios de crecimiento urbano propuesto por el grupo de expertos involucrados en el proyecto SIMURBAN. Se trata del escenario de "innovación y sostenibilidad", el cual pretende establecer un equilibrio entre los factores sociales, económicos y medioambientales, para generar un crecimiento sostenible con el territorio. En comparación con el crecimiento registrado en la Comunidad de Madrid en el periodo de calibración del modelo, el escenario reduce el crecimiento residencial un $17 \%$ y un $25 \%$ el crecimiento de superficie de uso productivo.

\section{MÉTODOS}

La propuesta metodológica se basa en la aplicación de un AS que permitirá determinar cómo los resultados se ven influenciados por los factores que interactúan en el modelo basado en AC. Para ello, se elaborarán diferentes versiones del modelo inicial, recogiendo en cada caso una combinación diferente de los factores que lo integran (tabla 1) pero simulando todos la misma cantidad de píxeles que se transformarán en zonas urbanas,. Así pues, las variaciones que podremos observar entre resultados atenderán únicamente a la localización de los crecimientos simulados. 
Tabla 1. Relación de factores implementados en cada modelo derivado del inicial. MODELOS M1 M2 M3 M4 M5 M6 M7 M8 PARÁMETROS Aleatoriedad

\begin{tabular}{|c|c|c|c|c|c|c|c|c|}
\hline MODELOS & \multirow{2}{*}{ Ml } & \multirow{2}{*}{ M2 } & \multirow{2}{*}{ M3 } & \multirow{2}{*}{ M4 } & \multirow{2}{*}{ M5 } & \multirow{2}{*}{ M6 } & \multirow{2}{*}{ M7 } & \multirow{2}{*}{ M8 } \\
\hline PARAMETROS & & & & & & & & \\
\hline Aleatoriedad & & & & & & & & $\mathrm{X}$ \\
\hline Vecindad & $\mathrm{X}$ & $\mathrm{X}$ & $\mathrm{X}$ & & $\mathrm{X}$ & & & $\mathrm{X}$ \\
\hline Accesibilidad & $\mathrm{x}$ & $\mathrm{X}$ & & $\mathrm{X}$ & & $\mathrm{X}$ & & $\mathrm{X}$ \\
\hline Aptitud & $\mathrm{X}$ & & $\mathrm{X}$ & $\mathrm{X}$ & & & $\mathrm{X}$ & $\mathrm{X}$ \\
\hline
\end{tabular}

Como se puede observar, la aleatoriedad únicamente se recoge en el modelo completo M8, dado que este factor no es estable. Estos modelos se ejecutarán y se compararán los resultados obtenidos para cada uso entre sí. En este caso el modelo que tomaremos como referencia será el M1 que incluye la totalidad de factores estables a excepción de la aleatoriedad (no estable).

\subsection{Métodos de Comparación de Mapas.}

Dentro de las diversas metodologías de comparación de mapas existentes, podemos decir que coexisten dos vertientes: la primera recoge los métodos más tradicionales, que se basan en la comparación píxel a píxel de los mapas; la segunda recoge otros métodos que, normalmente empleados para otros fines, son a nuestro juicio una fuente de información relevante para valorar las diferencias de patrones de crecimiento simulados y que además permiten una mayor flexibilidad de la evaluación de los resultados.

En este caso consideramos que el empleo de ambos tipos de procedimientos puede darnos una visión de conjunto detallada sobre la influencia de cada factor en los resultados y dotar a los mismos de mayor robustez. Como métodos tradicionales se ha decidido emplear la comparación visual y el porcentaje de coincidencia (ver tabla 2). En cuanto a los métodos empleados para otro tipo de fines, se ha decidido explorar las posibilidades que ofrecen la aplicación de áreas de influencia o buffers y la utilización de métricas espaciales o spatial metrics.

La comparación visual nos permitirá entender a grandes rasgos cómo varían los resultados ofrecidos por el modelo de forma general en función de los factores introducidos, así como observar tendencias espaciales fruto de los mismos. 
Por otro lado, el porcentaje de coincidencia es un índice calculado a partir de los píxeles coincidentes entre dos mapas, en función del total de píxeles simulados.

Los llamados buffers permiten generar zonas de influencia alrededor de los píxeles de nueva creación en el modelo que se tome como referencia en la comparación. Estas zonas se añaden a la superficie de crecimiento generada por el modelo en cuestión, de forma que al comparar los resultados de otro modelo con los de éste, se pueda determinar qué cantidad de píxeles caen dentro del área de influencia.

Estas áreas de influencia se han empleado con éxito en otros ámbitos, teniendo su origen en la utilización de la banda épsilon para verificar la exactitud de la cartografía de elementos lineales. Algunos autores han desarrollado procesos para medir la exactitud de la posición de elementos lineales, basados en la generación de este tipo de corredores (Goodchild y Hunter, 1997, Tveite y Langaas, 1999).

Es evidente que cuanto mayor sea el buffer, mayor será la coincidencia de resultados entre el modelo de referencia y el modelo a comparar. Por ello, y basándonos en el trabajo realizado por Tveite y Langaas (1999), se propone el empleo de dos buffera 1.000 y $2.000 \mathrm{~m}$ de distancia de los resultados generados por el modelo M1. Hemos de advertir que se compara solamente la superficie simulada de cada uso, no teniendo en cuenta la superficie preexistente en el año 2000, dado que aumentarían tanto los porcentajes de coincidencia, que sesgarían los resultados, impidiendo determinar correctamente las diferencias entre los distintos modelos ensayados.

Por otro lado las métricas espaciales (Herold et al, 2005) se han empleado con éxito en muy diversos ámbitos de estudio para estudiar los diferentes patrones de ocupación urbana (Irwin et al, 2007; Aguilera et al, 2011; Wu et al, 2011; Sun et al, 2013), e incluso para la comparación de los resultados de simulaciones (Petrov et al, 2009). Estas métricas permiten medir las características espaciales y morfológicas de las diferentes ocupaciones urbanas a través de un conjunto de valores numéricos. Dentro de las diferentes métricas que se pueden aplicar (ver Mcgarigal et al, 2002), se han escogido las más relevantes para el estudio. Así, el PN permitiría determinar el número de teselas de cada uso pudiendo valorarse su grado de fragmentación o agregación. EI SHAPE_MN por su parte permitirá conocer la forma de las teselas y finalmente el ENN_MN determinará la distancia media entre 
teselas, como una medida de la dispersión.

Las métricas señaladas se calcularán para la superficie total urbana resultado de la simulación, esto es, la superficie preexistente del uso en el año 2000 más la superficie simulada en el año 2020, ya que el interés de estas métricas es el de valorar los patrones de ocupación urbana resultantes y para ello necesitamos evaluar la superficie total del uso.

Tabla 2. Cuadro resumen de métodos de comparación empleados.

\begin{tabular}{|c|c|c|}
\hline \multicolumn{2}{|c|}{ METODO } & APLICACION \\
\hline \multicolumn{2}{|c|}{ Comparación Visual } & $\begin{array}{c}\text { Visualización de todos los mapas resultado de los modelos } \\
\text { y comparativa entre los mismos }\end{array}$ \\
\hline \multirow{2}{*}{ Porcentaje de Coincidencia } & $\begin{array}{c}\text { Porcentaje de pixeles del uso simulado coincidente entre } \\
\text { modelos }\end{array}$ \\
\cline { 2 - 3 } Buffer & 1000m & $\begin{array}{c}\text { Porcentaje de pixeles del uso simulado que caen dentro de } \\
\text { los buffer generados sobre los pixeles simulados por el } \\
\text { modelo de referencia }\end{array}$ \\
\hline \multirow{2}{*}{$\begin{array}{c}\text { Métricas } \\
\text { espaciales }\end{array}$} & SHAPE_MN & $\begin{array}{c}\text { Número de teselas (pixeles contiguos del mismo uso), nos } \\
\text { indica la fragmentación/agregación del uso forma media, aporta información en relación a la } \\
\text { forma de las teselas. }\end{array}$ \\
\cline { 2 - 3 } & ENN_MN & $\begin{array}{c}\text { Distancia media entre teselas, señala la dispersión o } \\
\text { concentración de un uso. }\end{array}$ \\
\hline
\end{tabular}

\subsection{Mapa de las zonas más veces seleccionadas para el desarrollo urbano}

Finalmente es posible localizar aquellas zonas del territorio que sistemáticamente, e independientemente de las variaciones realizadas en el modelo, son seleccionadas como nuevas zonas a urbanizar en un mayor número de simulaciones. Se trataría, por tanto, de los resultados más estables y robustos, y, en cierto modo, nos estaría indicando que son las zonas que poseen una mayor tendencia a ser urbanizadas. Para ello se utilizará la cartografía de los píxeles más veces seleccionados (Lodwick, 1989; Lowry et al., 1995; Canters et al. 2002, Gómez Delgado y Bosque Sendra, 2004). De esta forma, un mapa que refleje los píxeles más repetidos en las simulaciones podrá derivar en una mejora significativa de la planificación urbana, disminuyendo el riesgo y la incertidumbre asociada a la toma de decisiones en este ámbito concreto. 


\section{RESULTADOS Y DISCUSIÓN}

\subsection{Comparación visual.}

Una vez ejecutados los modelos, se han obtenido para cada uno dos mapas, uno que muestra la situación tras la simulación del uso residencial para el año 2020 y otro que refleja la situación para esa misma fecha del uso productivo. En la figura 4 aparecen los resultados más representativos.

Figura 4. Detalle de algunos de los resultados más relevantes generados los modelos derivados del inicial.

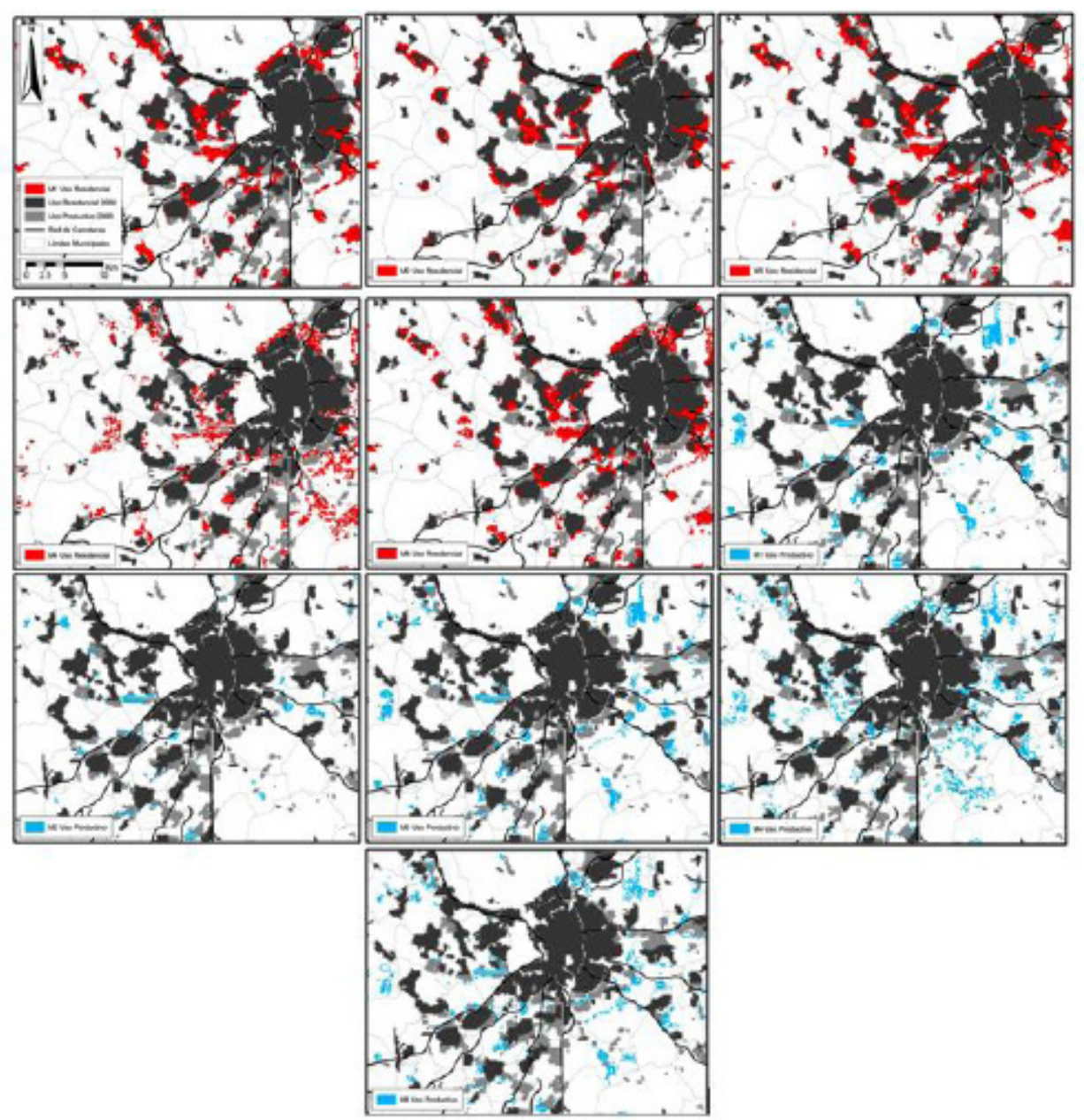


Podemos constatar que los modelos M8 y M1 son muy similares (con algunas leves diferencias en el uso productivo), estando sus diferencias principales en localizaciones aisladas generadas principalmente por la aleatoriedad. Además M3 se muestra bastante similar a estos modelos lo que puede indicar que la accesibilidad no está teniendo un efecto especialmente relevante en los resultados.

Por otro lado, se observa que M2 se diferencia más de los dos modelos anteriores (M8 y M1), adoptando en el caso del uso residencial otro patrón de crecimiento (tendencia circular), mientras que para el uso productivo las grandes formaciones de nuevas zonas comerciales e industriales dan lugar a emplazamientos más diseminados y fragmentados. En el caso de M4, los resultados parecen estar mucho más fragmentados que $\mathrm{M} 1$, dado que se elimina la vecindad y el modelo tiende a localizar los nuevos crecimientos en las zonas con mayor aptitud.

Con esta primera aproximación visual es posible hacerse una idea general de las principales diferencias entre los modelos, pero será necesario algún tipo de apoyo matemático que complemente esta información y nos permita cuantificar las diferencias diversos resultados.

\subsection{Porcentaje de coincidencia y Buffers.}

A continuación se muestran los porcentajes de coincidencia derivados de la comparación neta de cada uno de los resultados de los modelos con el modelo M1 de referencia. Además se recoge el porcentaje de coincidencia entre el área de influencia generado por el buffer $(1.000$ y $2.000 \mathrm{~m})$ del modelo de referencia y los resultados del resto de modelos (tabla 3 ).

Es necesario advertir que la superficie preexistente en el año 2000 en los dos usos estudiados es del orden de entre 2 y 3 veces superior en comparación con la superficie generada por la simulación. Dada esta situación, la comparación como se indicó en el apartado 3 se realizará teniendo en cuenta solamente la superficie simulada para 2020. 
Tabla 3. Porcentajes de coincidencia de los resultados generados entre los modelos derivados y M1, donde PC es el porcentaje de coincidencia, B1000 es el buffer a $1.000 \mathrm{~m}$ y B2000 es el buffer a $2.000 \mathrm{~m}$.

\begin{tabular}{|c|c|c|c|c|c|c|}
\hline Model0 & PC & PC+B1000 & PC+B2000 & PC & PC+B1000 & PC+B2000 \\
\hline M2 & 37,12 & 69,56 & 84,45 & 40,71 & 78,39 & 84,88 \\
\hline M3 & 90,52 & 99,67 & 99,96 & 93,46 & 99,57 & 99,65 \\
\hline M4 & 40,00 & 69,04 & 81,68 & 22,56 & 64,96 & 82,99 \\
\hline M5 & 37,03 & 69,56 & 84,48 & 40,79 & 78,62 & 85,04 \\
\hline M6 & 5,59 & 36,68 & 54,54 & 21,73 & 51,26 & 68,90 \\
\hline M7 & 39,76 & 68,67 & 81,18 & 22,32 & 67,09 & 83,27 \\
\hline M8 & 81,14 & 98,17 & 99,50 & 74,92 & 97,44 & 98,94 \\
\hline
\end{tabular}

El modelo que presenta mayor similitud con M1 es M3, donde la coincidencia alcanza porcentajes superiores al $90 \%$ en ambos usos. Esto parece indicar que la accesibilidad no está influyendo excesivamente en los resultados, como mostraba la comparación visual. Además se demuestra que los resultados que no coinciden de forma directa se encuentran dentro del primer buffer, es decir, muy próximos a los resultados de referencia (más de $99,5 \%$ de coincidencia).

En segundo lugar, el modelo M8 es el segundo con mayor similitud espacial con M1. La aplicación del primer buffer, nos señala que los resultados debidos a la aleatoriedad no coinciden espacialmente con los de M1 píxel a píxel, pero sí a una cierta distancia de estos $(1.000 \mathrm{~m})$. Podemos afirmar pues, que la aleatoriedad localiza los resultados en las proximidades de los ofrecidos por M1, formando el resto de píxeles que no entran en el segundo buffer un tipo de crecimiento aislado. Por otro lado el M2, donde se elimina la aptitud, alcanza una coincidencia espacial del $40 \%$ aproximadamente en ambos usos, valores que aumentan hasta colocarse en el segundo buffer entorno al $85 \%$. Se comprueba que al no incluir este factor, los píxeles de nuevo crecimiento se situán lejos de los preexistentes. Estos resultados son prácticamente idénticos a los recogidos en la comparación con M5. Además, en el caso de M4 ocurre algo similar, siendo los resultados muy parecidos. El efecto es un poco más evidente para el uso productivo. En la misma línea de resultados se encuentra el modelo M7.

En cuanto al modelo M6, al incluir solamente la aleatoriedad, se demuestra 
la importante influencia de los parámetros de vecindad y aptitud en los resultados del modelo.

\subsection{Métricas espaciales (Spatial Metrics)}

Los modelos que más se asemejan el patrón de crecimiento del modelo de referencia M1 son M3 y M8. Se comprueba que la aleatoriedad o la accesibilidad poco influyen en la definición del patrón de crecimiento asociado a este escenario. La única diferencia remarcable puede estar en la distancia entre teselas, dado que la aleatoriedad daba lugar a crecimientos aislados, por ello el ENN_MN para M8 dista levemente del M1.

Por otro lado, se aprecia cómo la aptitud genera resultados más fragmentados en el uso productivo, por ello su eliminación hace que desciendan los valores de PN en M2 con respecto a M1; las formas son más compactas y se disminuye la dispersión, dados los valores altos en ENN_MN. Para el uso productivo, las formas son más complejas, pero la dispersión y fragmentación/agregación son similares a las de M1.

Figura 5. Valores obtenidos para las métricas espaciales en los modelos empleados.

En azul para el modelo productivo y rojo el residencial. La línea discontinua recoge el valor para el modelo de referencia M1

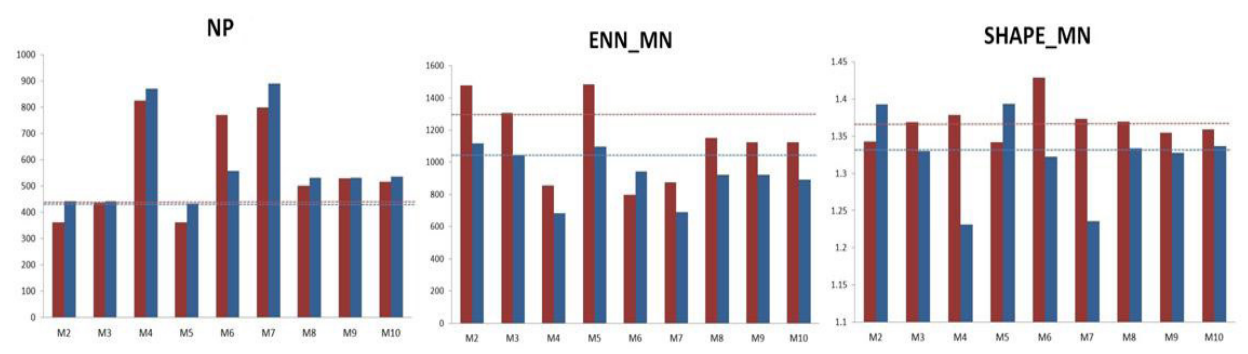

En el caso de la vecindad, su exclusión en M4 puede ser el más llamativo por las diferencias en los resultados en ambos usos. La fragmentación es muy superior al modelo de referencia (del orden del doble) Las formas del uso productivo son más próximas a círculos, registrándose valores muy próximos a la unidad en el índice SHAPE_MN. Además, al aumentar la fragmentación, disminuye la distancia media entre teselas, (ver ENN_MN). Luego se puede decir que la vecindad fomenta la agregación, lo que resulta algo lógico, dada la naturaleza de este parámetro. 
Además genera resultados más dispersos, dado que su eliminación crea teselas más próximas. La eliminación conjunta de vecindad y accesibilidad (M7) aporta los mismos resultados prácticamente que M4, mostrando otra vez la poca influencia de la accesibilidad. La acción conjunta entre aptitud y vecindad (eliminados en M6) muestra los resultados más dispares.

\subsection{Localización de los resultados más estables a partir de la cartografía de los píxeles más veces seleccionados.}

En la figura 6 se recogen los emplazamientos de nuevo crecimiento generados por los 8 modelos, en función del número de veces que estos han sido seleccionados en dichos modelos. En el caso del uso residencial parece existir una fuerte tendencia en el modelo a localizar en el entorno más inmediato del núcleo urbano de Madrid los nuevos emplazamientos, además de tender a ocupar los espacios intersticiales entre los diferentes núcleos urbanos próximos entre sí. Es ahí donde se recogen los valores más altos de coincidencia (entre 5 y 8 veces seleccionados).

Por otro lado, el uso productivo sigue una tendencia diferente, al generarse una menor cantidad de crecimiento para 2020 en comparación con el residencial. Para este uso observamos pocas zonas seleccionadas por todos o casi todos los modelos, estando el principal punto común en ciertas zonas cercanas a otros núcleos industriales y/o comerciales ya existentes y próximos o en línea con las vías de comunicación principales.

Figura 6. Zonas más veces recogidas por los modelos como resultado simulado a futuro
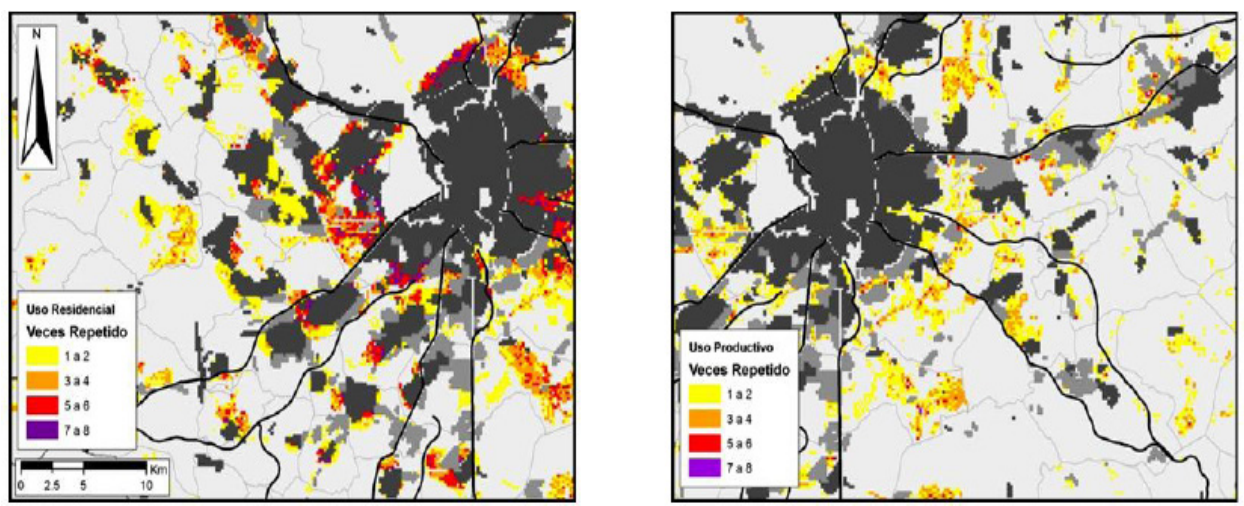


\section{CONCLUSIONES}

Pensamos que en el presente trabajo ha quedado demostrado que es necesario y posible establecer propuestas alternativas de validación de aquellos modelos que pretenden realizar simulaciones futuras plausibles y/o deseables, y cuyos resultados no pueden ser contrastados y verificados con datos reales. La aplicación de un sencillo AS ha aportado información interesante y valiosa sobre la influencia de cada uno de los factores incluidos en el modelo sobre la estabilidad de sus resultados. Por otra parte nos ha permitido detectar posibles deficiencias en el tratamiento y modelación de alguno de estos factores. Concretamente parece que el factor de accesibilidad no está resultando apenas influyente, pues su eliminación en el modelo no provoca cambios sustanciales en los resultados (M3). Sin embargo, se trata de un factor que tradicionalmente ha sido determinante en la creación de nuevos desarrollos urbanos. Será necesario revisar el tratamiento que de él se ha hecho para que resulte de mayor influencia en los resultados, tal y como sería esperable.

En cuanto a las propuestas para llevar a cabo la evaluación de los resultados del AS, la aplicación de la metodología tradicional de comparación de mapas (comparación visual y coincidencia píxel a píxel) permite tener una visión general y valiosa de las diferencias a grandes rasgos entre los modelos presentados. Sin embargo, la aplicación de áreas de influencia o buffers demuestra que la comparación píxel a píxel puede ser demasiado rígida y poco apropiada para evaluar los resultados de la simulación de este tipo de fenómenos (dinámica urbana), y que resulta razonable incorporar cierta flexibilidad en la coincidencia de los resultados. Además, las métricas espaciales han demostrado que su aplicación y comparación entre modelos puede proporcionarnos claves interesantes para entender cómo varían los patrones de crecimiento en función de los factores introducidos en el modelo.

Por otro lado se ha comprobado que dependiendo del método de comparación de mapas seleccionado es aconsejable el uso de la superficie simulada solamente o la total en la comparación de los resultados. Así, por ejemplo en el caso concreto de las métricas espaciales, es necesario emplear la superficie total para conocer los patrones resultado de la simulación.

Por último la cartografía de los píxeles más veces seleccionados nos ha permitido identificar de manera sencilla aquellas zonas con más tendencia a de- 
sarrollarse según el modelo y escenario planteados. Es importante que estos emplazamientos sean tenidos en cuenta a la hora de realizar nuevas planificaciones urbanas en este entorno, dado que parecen ser las más robustas para albergar los nuevos usos simulados por el modelo. En este caso concreto, hemos podido comprobar que los resultados para el uso residencial son, en términos generales, bastante robustos y estables. Sin embargo, la localización de usos productivos resulta más incierta, lo que debería obligarnos a revisar la estructura de este modelo y la modelación de las variables que forman parte del mismo y prestar especial atención a la hora de planificar estas zonas.

\section{BIBLIOGRAFÍA}

- Aguilera Benavente, F., 2006: "Predicción del crecimiento urbano mediante sistemas de información geográfica y modelos basados en autómatas celulares". GeoFocus, 6: pp. 81-112.

- Aguilera Benavente, F. y Talavera García, R., (2009): "Valoración de escenarios futuros a través de la conectividad del paisaje", Observatorio Medioambiental, 12, pp. 17-36.

- Aguilera Benavente F, Plata Rocha W, Bosque Sendra J, Gómez Delgado M, 2009: "Hacia la simulación de escenarios futuros de demanda de ocupación de suelo en ámbitos metropolitanos", in Proceedings of II Internacional Conference on Sustainabiltiy Measurement and Modelling, 1-21, Terrassa, España.

- Aguilera Benavente, F.; Valenzuela Montes, L.M. y Bosque Sendra, J., (2010): "Simulación de escenarios futuros en la aglomeración urbana de Granada a través de modelos basados en Autómatas Celulares", Boletín de la Asociación de Geógrafos Españoles, 54, pp. 271-300.

- Aguilera Benavente, F.; Valenzuela Montes, L.M.; y Botequilha-Leitão, A., (2011): "Landscape metrics in the analysis of urban land use patterns: A case study in a Spanish metropolitana area", Landscape and Urban Planning, 99, pp. 226-238.

- Barredo, J.; Kasanko, M.; McCornick, M. y Lavalle, C., (2003): "Modelling dynamic spatial processes: simulation of urban future scenarios through celular automata", Landscape and Urban Planning, 64, pp. 145-160. 
- Barredo, J.I.; Demicheli, L.; Lavalle, C.; Kasanko, M. y McCormick, N., (2004): "Modelling future urban scenarios in developing countries: an application case study in Lagos, Nigeria", Environment and Planning B: Planning and Design, 32, pp 65-84.

- Batty, M. y Xie, Y. (1997): "Possible Urban Automata", en Besussi, E y Cecchini, A (Ed): Artificial Worlds and Urban Studies. Venezia, DAEST, pp 153-164.

- Benenson, I. y Torrens, P., (2004): GEOSIMULATION: Automata-based modelling of urban phenomena. Hoboken, NJ: John Wiley \& Sons.

- erdoulay, V. (2009). La historia de la Geografía en el desafío de la prospectiva. Boletín de la A.G.E.51: 9-23

- Berling-Wolf, S. y Wu, J., (2004): "Modelling urban landscape dynamics: A case study in Phoenix, USA", Urban Ecosystems, 7, pp. 215-240.

- Burriel, E.L. (2011). Subversion of land-use plans and the housing bubble in Spain, Urban Research \& Practice, Vol.4, No.3, pp. 232-249.

- Canters, F. y Genst, W. De y Dufourmont, H. (2002): "Assessing effects of input uncertainty in structural landscape classification", International Journal of Geographical Information Science, Vol.16, No.2, pp. 129-149.

- Dreborg 2004. Scenarios and Structural Uncertainty: Explorations in the Field of Sustainable Transport. PhD thesis. Stockholm: Royal Institute of Technology.

- García, A.M.; Santé, I.; Crecente, R. y Miranda, D. (2011): "An analysis of the effect of the stochastic component of urban cellular automata models", Computers, Environment and Urban Systems, 35, pp. 286-296.

- Gómez Delgado, M. y Bosque Sendra, J. (2004): "Aplicación de análisis de incertidumbre como método de validación y control del riesgo en la toma de decisiones", GeoFocus (Artículos), nº 4, p. 179-208. ISSN: 1578-5157

- Gómez Delgado, M y Bosque Sendra, J. (2009): "Validation of GIS-performed analysis", Geoinformatics for Natural Resource Management, NOVA Science Publisher (ISBN 978-160692-211-8), pp. 559-571, New York (USA) 
- Goodchild, M.F. y Hunter, G.J. (1997): "A simple positional accuracy measure for linear features", International Journal of Geographical Information Science, Vol.11, No.3, pp. 299-306.

- Herold, M; Couclelis, H, y Clarke K.C. (2005) "The role of spatial metrics in the analysis and modelling of urban land use change". Computer and Environment Systems, n² 29, p. 369-399.

- Irwin, E.G. y Bockstael, N.E. (2007): "The evolution of urban sprawl: Evidence of spatial heterogeneity and increasing land fragmentation", Proc Natl Acad Sci U S A. December 26; 104(52): 20672-20677.

- Kocabas, V., y Dragicevic, S., (2006): "Assessing cellular automata model behavior using a sensitivity analysis approach", Computers, Environment and Urban Systems, 30, pp. 921-953.

- Lodwick, W. (1989): "Developing confidence limits on errors of suitability analysis in GIS", en Goodchild, M.F. y Gopal, S. (Eds.): The Accuracy of Spatial Databases. London, Taylor \& Francis, pp.69-78.

- $\quad$ Lowry, J.H; Miller, H.J. y Hepner, G.F. (1995): “A GIS-based sensitivity analysis of community vulnerability to hazardous contaminants on the Mexico/US border", Photogrammetric Engineering and Remote Sensing, Vol. 61, No.11, pp. 1347-1359.

- Ménard A. ; Marceau, D.J. (2005): "Exploration of spatial scale sensitivity in geographic cellular automata". Environment and Plan. B, 32 (2005), pp. 693-714

- OSE, Observatorio de la Sostenibilidad en España (2006): Cambios de ocupación del suelo en España. Implicaciones para la sostenibilidad. Alcalá de Henares, OSE.

- Paegelow, M. y Camacho, M.T (2008): Modelling Environmental Dynamics. Berlin, Springer-Verlag.

- Pan, X.Z. y Zhao, Q.G. (2011): "Measurement of urbanization process and the paddy soil loss in Yixing city, China between 1949 and 2000". CATENA. Volume 69, Issue 1, 16 January 2007, Pages 65-73 
- Petrov, L., Lavalle, C., y Kasanko, M. (2009): "Urban land use scenarios for a tourist region in Europe: Applying the MOLAND model to Algarve, Portugal", Landscape and Urban Planning, 92, pp. 10-23

- Plata Rocha, W.; Gómez Delgado, M. y Bosque Sendra, J. (2010): "Desarrollo de modelos de crecimiento urbano óptimo para la Comunidad de Madrid aplicando Métodos de Evaluación Multicriterio y Sistemas de Información Geográfica"; GeoFocus (Artículos), nº 10, p. 103-134. ISSN: 1578-5157.

- Plata Rocha, W., Gómez Delgado, M. y Bosque Sendra, J. 2011. "Simulating urban growth scenarios using GIS and Multicriteria Evaluation techniques: a case study of the Madrid Region, Spain", Environment and Planning B: Planning and Design, Vol.38, 1012-1031.

- Qureshi, M.E., 1999. Development and Implementation of a Decision Support Process for Sustainable Catchment Management. PhD thesis, The University of Queensland, Brisbane.

- Santé, I., García, A.M., Miranda, D. y Crescente, R., (2010): "Cellular autómata models for the simulation of real world urban processes: A review and analysis", Landscape and Urban Planning, 96, pp. 108-122.

- Saltelli, A., Chan, K. y Scott, E.M., (2000): Sensitivity Analysis. Chichester, John Wiley \& sons.

- Saltelli, A, M. Ratto, T. Andres, F. Campolongo, J. Cariboni, D. Gatelli, M. Saisana, S. Tarantola (2008): Global Sensitivity Analysis. The Primer; John Wiley and Sons.

- Sun, C.; Wu, Z.; Lv, Z;; Yao, N. y Wei, J (2013): "Quantifying different types of urban growth and the change dynamic in Guangzhou using multi-temporal remote sensing data" International Journal of Applied Earth Observation and Geoinformation. Vol. 21. Pp. 409-417

- Tveite, H. y Langass, S. (1999): "Accuracy assessments method for geographical line data sets based on buffering", International Journal of Geographical Information Science, Vol.13, No.1, pp. 27-47. 
- Torrens, P.M., (2000): "How cellular models of urban systems work". London, WP-28. Centre for Advanced Spatial Analysis (CASA), University College London.

- White, R., Engelen, G., y Uljee, I., (1997): "The use of constrained cellular automata for high resolution modelling of urban land use dynamics", Environment and Planning B: Planning and Design, 24, pp. 323-343.

- Wu, J.; Jenerette, G.D.; Buyantuyev, A. y Redman, C.L. (2011): "Quantifying spatiotemporal patterns of urbanization: The case of the two fastest growing metropolitan regions in the United States" Ecologycal Complexity; vol 8.pp. 1-8. 